\title{
Endometriosis and Its Relationship with Depression
}

\section{Roberta Furtado Stivanin Rachid Novais ${ }^{1 *}$, Bartolomeu Expedito da Câmara-França ${ }^{1}$, Ricardo Bassil Lasmar'1, Bernardo Portugal Lasmar²}

\author{
${ }^{1}$ Universidade Federal Fluminense (UFF), Rio de Janeiro (RJ), Brazil \\ ${ }^{2}$ Universidade Estácio de Sá (UNESA), Rio de Janeiro, Brazil \\ Email: ^rsrn1981@gmail.com, camarafranca@oi.com.br, ricardo@lasmar.com.br, bernardo@lasmar.com.br
}

How to cite this paper: Novais, R.F.S.R., da Câmara-França, B.E., Lasmar, R.B. and Lasmar, B.P. (2018) Endometriosis and Its Relationship with Depression. International Journal of Clinical Medicine, 9, 71-78. https://doi.org/10.4236/ijcm.2018.92008

Received: January 22, 2018

Accepted: February 5, 2018

Published: February 8, 2018

Copyright $\odot 2018$ by authors and Scientific Research Publishing Inc. This work is licensed under the Creative Commons Attribution International License (CC BY 4.0).

http://creativecommons.org/licenses/by/4.0/ cc) (i) Open Access

\begin{abstract}
INTRODUCTION: There is evidence that the physical and psychic pain resulting from endometriosis is responsible for depression, and that one disease would complicate the other. OBJECTIVE: The objective of the study is to detect depressive symptoms in women diagnosed with endometriosis, comparing them with those without the disease, and to evaluate if there is a relationship between the presence of current depressive symptoms and in childhood/adolescence. METHODS: Were done Beck's Depression Inventory for adults and an adaptation of the infant, both self-applied. RESULTS: Of the women with endometriosis, $66 \%$ had symptoms of depression in the Beck's Inventory, while in the control group, $58 \%$ had some degree of depression, but Fisher's exact test showed that it was not possible to identify the relationship between endometriosis and depression $(p=0.423)$. CONCLUSION: The entity endometriosis has no relation to present or past depressive symptoms. When depressed, women with endometriosis tend to have mild symptoms for reasons not yet known, requiring further research on the subject.
\end{abstract}

\section{Keywords}

Endometriosis, Depression, Personality Inventory

\section{Introduction}

Endometriosis is defined as the presence of tissue similar to endometrium, with glands and stroma, outside the cavity of the uterus [1]. Its incidence is $10 \%$ $15 \%$ of women of all groups, ethnic and social, reproductive and immediate postmenopausal stages [2], which would confirm the hypothesis of estrogen dependence [3]. The difficulty of assessing the prevalence is dependent on the sur- 
gical confirmation [4].

It is found in $25 \%$ of women with pelvic pain and $20 \%$ of women with infertility undergoing laparoscopy, endometriosis is considered the main responsible for these two female complaints when at reproductive age [5].

Of the clinical manifestations, besides chronic pelvic pain, are mentioned: severe dysmenorrhea, dyspareunia deep, pain related to the intestinal and urinary functions, mainly dyschezia and dysuria in menstrual period [3]. It can also be asymptomatic, with a prevalence of $3 \%$ to $43 \%$ [5].

Endometriosis has been linked to increased symptoms of mental origin, such as depression, anxiety, and increased stress [6], perhaps due to the fact that the high prevalence of depression in women with endometriosis is due to both cognitive impairment and alteration of their vital functions, thus reducing their quality of life [2].

One of the most studied psychological factors in people with chronic pain is depression, regardless of the disease associated with this symptom [7], and therefore, there is evidence that the physical and psychic pain resulting from endometriosis is responsible for the depression, and that one disease aggravates the other, and there is no consensus on the temporal issue when defining which preexisting condition [8].

Depression is defined as a common mental disorder characterized by loss of interest, lack of pleasure, fluctuations between feelings of guilt and low self-esteem, as well as sleep or appetite disorders. There is also a feeling of tiredness and lack of concentration [8] [9].

The objective of this study is to evaluate the relationship between endometriosis and depression, from questionnaires self-applied, submitted to patients attended at the outpatient clinic of the Hospital Universitário Antônio Pedro of the Universidade Federal Fluminense (HUAP).

\section{Methods}

This is an observational, analytical, cross-sectional, case-control, prospective study in patients with and without endometriosis from the Gynecology Outpatient Clinic of the Hospital Universitário Antônio Pedro (HUAP), from September 18, 2014 to July 30, 2015.

Approved by Ethics and Research Commission of the Faculty of Medicine of the Universidade Federal Fluminense-FM/UFF/HU.

Were evaluated 103 patients, 53 cases with endometriosis (Group 1) and 50 without endometriosis (Group 2). Estimated number based on the significant percentage of $10.4 \%$ of the Brazilian population that presented depression in 2011 [10].

The 53 patients were treated at the endometriosis outpatient clinic and 50 controls were patients of family planning clinic of the HUAP gynecology service.

Inclusion criteria of the case group were patients of reproductive age or perimenopause, with anatomopathological confirmation of pelvic endometriosis, at- 
tended at the outpatient clinic of Gynecology of HUAP. Exclusion criteria: women with infectious diseases or degenerative diseases not related to endometriosis, post-operative of any diseases, except endometriosis, in radiotherapeutic or chemotherapeutic treatment or who did not agree to participate in the research.

The inclusion criteria for patients in the control group were from the planning clinic family of HUAP, being of reproductive age or perimenopause, candidates for tubal ligation, guaranteeing previous fertility, who denied pelvic pain, disabling dysmenorrhea, dyschezia, dyspareunia, pain with changes in bowel habit during menstruation, menstrual dysuria or recurrent urinary tract infection. Exclusion criteria are the same as in case group, women with infectious diseases or degenerative diseases not related to endometriosis, post-operative of any diseases, in radiotherapeutic or chemotherapeutic treatment or who did not agree to participate in the research. In addition, if they presented imaging tests proving endometriosis.

Even though these data would not guarantee the absence of endometriosis in this group, we could assume that the disease would be less likely. Those that were submitted to ligature until the end of the study did not present endometriosis. All imaging tests were evaluated (Table 1).

Due to information difficulty of the control group, it was not possible to compare clinical, psychological and social characteristics, body mass index, comorbidities, use of medications, previous pathological history, family and labor relations, economic income.

The 103 patients were evaluated by two self-administered questionnaires, at the outpatient clinic, without any interference from the researcher.

First, the Beck Depression Inventory (BDI), a measurement instrument to self evaluate the state of depression. The classifications of scores indicative for depression are: normal (0 - 9), mild (10 - 15), mild to moderate (16 - 19), moderate to severe $(20-29)$, severe $(30-63)$.

The other self-administered questionnaire was an adaptation of the Children's Depression Inventory (CDI), which originally proposed measuring depressive symptoms in young people aged 7 to 17 years, either through self-application or

Table 1. Characteristics of the patients in the HUAP family planning outpatient control group.

They have already shown their fertility.

They denied dyspareunia.

They denied disabling dysmenorrheal.

They denied pain with changes in bowel habit during menstruation.

They denied menstrual dysuria or recurrent urinary tract infection.

They did not present an image of ovarian cyst (endometrioma) or suggestive of endometriosis in image examination (transvaginal ultrasonography). 
by responses from informants (parents, teachers or friends), and can be applied individually or collectively. A cut-off point of 18 was established, a score equal to or greater than 18 would be considered suspected of having a depressive disorder.

The statistical method used was the chi-square test for intergroup analysis. For the study were separated patients with and without endometriosis, with in no signs of current and/or past depression. Fisher's exact test was used to evaluate this association. The program used was SPSS for Windows and the value was $\mathrm{p}<0.05$.

\section{Results}

Of the 103 patients, 53 (51.5\%) had histopathological diagnosis of endometriosis (case) and 50 (48.5\%) with proven natural fertility, without endometriosis diagnosis (control). The age group ranged from 15 to 49 years, with the average age being 33.4 years.

Regarding the level of education of the women investigated, 5 (4.9\%) reported incomplete elementary school, 8 (7.8\%) complete elementary school, 12 (11.7\%) incomplete high school, 46 (44.7\%) completed high school, 6 (5.8\%) college degree incomplete and $26(25.2 \%)$ complete (Table 2).

Of the total number of interviewees, 9 (8.7\%) reported using psychotropic drugs, mainly benzodiazepines. The use of hormones was claimed by 67 (65\%) of the participants, including oral contraceptives and combined injections of estrogen and progesterone, oral and injectable progesterone contraceptives and oral progesterone, for the treatment of endometriosis pain. In the case group, 3 (5.7\%) women reported oral progesterone use and 2 (3.8\%) injectables. None reported current use of Gonadotropin-Releasing Hormone Analogues or use of a progesterone intrauterine device.

In the studied population, according to the Beck Inventory, there were scores compatible with depression in $64(62.1 \%)$ of the women, being $27(26.2 \%)$ in a mild degree, 6 (5.8) mild to moderate, 16 (15.5\%) moderate to severe and 15 (14.6\%) severe depression. No current depressive grade was found in 39 (37.9\%) of the respondents (Table 3 ).

Table 2. Frequency of education in the study population.

\begin{tabular}{ccc}
\hline Degree of education & Frequency (n) & Percentage (\%) \\
\hline Complete high school & 46 & 44.7 \\
Graduated & 26 & 25.2 \\
Incomplete high school & 12 & 11.7 \\
Complete primary education & 8 & 7.8 \\
Incomplete higher & 6 & 5.8 \\
Incomplete elementary school & 5 & 4.9 \\
Total & 103 & 100.0 \\
\hline
\end{tabular}


Table 3. Degree of current depressive symptoms in the study population.

\begin{tabular}{ccc}
\hline Degree of current depressive symptoms & $(\mathbf{n})$ & (\%) \\
\hline No current depressive symptoms & 39 & 37.9 \\
Mild & 27 & 26.2 \\
Mild to moderate & 6 & 5.8 \\
Moderate to severe & 16 & 15.5 \\
Severe & 15 & 14.6 \\
Total & 103 & 100.0 \\
\hline
\end{tabular}

Table 4. Degree of current depressive symptoms by beck inventory.

\begin{tabular}{ccccccc}
\hline & \multicolumn{4}{c}{ Degree of current depressive symptoms n (\%) } & \multirow{2}{*}{ Total } \\
\cline { 2 - 6 } Group & No symptoms & Mild & $\begin{array}{c}\text { Mild to } \\
\text { moderate }\end{array}$ & $\begin{array}{c}\text { Moderate } \\
\text { to severe }\end{array}$ & Severe & \\
\hline Case & $18(34)$ & $20(37.7)$ & $4(7.5)$ & $6(11.3)$ & $5(9.4)$ & $53(100.0)$ \\
Control & $21(42)$ & $7(14)$ & $2(4)$ & $10(20)$ & $10(20)$ & $50(100.0)$ \\
Total & $39(37.8)$ & $27(26.2)$ & $6(5.8)$ & $16(15.5)$ & $15(14.5)$ & $103(100.0)$ \\
\hline
\end{tabular}

${ }^{*}$ Fisher's test; $\mathrm{p}=0.042$.

In an evaluation by Fisher's exact test there is an association between the degrees of current depressive symptoms obtained in women in both the case group and the control group $(\mathrm{p}=0.042)$, that is, women with endometriosis when depressive tend to have mild symptoms, whereas in those women who do not have the disease, the degrees of depression are higher (Table 4).

The Beck Inventory detected depressive symptoms in 35 (66\%) women with endometriosis. Of these, 20 (37.7\%) were found to be mild, in 4 (7.5\%) mild to moderate, $6(11.3 \%)$ moderate to severe depression and in severely depressed 5 (9.4\%). Patients interviewed with no depressant evidence accounted for 18 (34\%) of the case group.

Among women without endometriosis, 29 (58\%) presented some degree of depression due to the Beck Inventory. Of these, 7 (14\%) presented mild depression, 2 (4\%) mild to moderate, 10 (20\%) depression moderate to severe and 10 (20\%) severe. In 21 (42\%) investigated from the control group, no identified depressive symptoms by the Beck Invetory.

Fisher's exact test showed no statistically significant difference between the groups regarding the presence of depressive symptoms (Table 5).

For the adjusted CDI, depressive symptoms were found in 17 (16.5\%) patients in childhood and adolescence, while $86(83.5 \%)$ did not present such symptoms by the questionnaire.

In the group with confirmed endometriosis, 7 (13.2\%) surveyed showed current and past depressive symptoms. At the control, 5 (10\%) exhibited depressive symptoms in the past and present. However, there was no relationship between the three variables $(\mathrm{p}=0.620$ ), being endometriosis, current and past depressive 
Table 5. Current depressive symptoms in the groups studied.

\begin{tabular}{cccc}
\hline \multirow{2}{*}{ Group } & \multicolumn{2}{c}{ Current depressive symptoms $\mathbf{n}(\%)$} & \multirow{2}{*}{ Total } \\
\cline { 2 - 3 } & Yes & No & \\
\hline Case & $35(66)$ & $18(34)$ & $53(100)$ \\
Control & $29(58)$ & $21(42)$ & $50(100)$ \\
\hline
\end{tabular}

${ }^{*}$ Fisher's test; $\mathrm{p}=0.423$.

Table 6. Current and past depressive symptoms in the groups studied.

\begin{tabular}{cccc}
\hline \multicolumn{2}{c}{ Symptoms of depression } & \multicolumn{2}{c}{ Group n (\%) } \\
\hline Current & Past & Case & Control \\
\hline Yes & Yes & $7(13.2)$ & $5(10)$ \\
Yes & No & $28(52.8)$ & $24(48)$ \\
No & Yes & $2(3.8)$ & $3(6)$ \\
No & No & $16(30.2)$ & $18(36)$ \\
\hline
\end{tabular}

${ }^{*}$ Fisher's test; $\mathrm{p}=0.620$.

symptoms, without association (Table 6).

Pain was not used as an absolute criterion of inclusion in the case group, for reasons: better comparison with the control, since in this group there are no pelvic pain complaints; to ascertain the endometriotic disease as entity, that is, the stigma of the diagnosis, regardless of the permanence of the pain.

\section{Discussion}

Even with a small representative sample, the majority of the population studied (62.1\%) have depressive symptoms, perhaps due to underdiagnosis of the disease, caused by physicians who may lack training and time, disbelief regarding the effectiveness of treatment, distinguishing only physical symptoms and identifying the symptoms of depression as an "understandable reaction", and by patients, by the prejudice in recognizing themselves depressed and by disbelief regarding treatment. Thus, current depression was present in $66 \%$ of patients with endometriosis and in $58 \%$ of those without endometriosis, with no significant statistical difference.

Women with endometriosis have pain symptoms that often apparently do not relate to the stage of the disease and this is due to some psychological factors such as coping, emotional inhibition, depression, and anxiety. Coping is the mechanisms and strategies that the individual uses to deal with stressful situations. There are positive correlations between coping and depression/ anxiety and between severity of subjective pain and psychosocial development [11] [12].

Using Fisher's exact test, there was a significant $(p=0.042)$ difference between the current depression level according to the Beck Inventory in the study population, that is, there was an association between endometriosis and mild depressive symptoms, $37.7 \%$. The increased frequency of mild depression may be re- 
lated to coping, to a possible conformism because they are subject to indeterminate therapy without, above all, the certainty of cure, and it means that those who today have mild depression may have previously been treated for moderate and/or severe depression.

For the adapted questionnaire of childhood depression, we have the imprecision of the participants' memory, as well as the constraint in honestly exposing the feelings and the symptoms of current depression in the women who presented high values in the Beck Depression Inventory, since everything is evaluated by the optics of negativism.

Of the women with current and past depressive symptoms, $13.2 \%$ belong to the case group and $10 \%$ to the control, without significant statistical difference $(p=0.620)$, therefore no association between endometriosis and present or previous depressive symptoms.

Thus, according to the foregoing, endometriosis has no relation to symptoms present or past depressive disorders and, when depressed, women with endometriosis tend to present mild symptoms for reasons not yet known, requiring further research on the subject.

The population of this study is composed of patients with depressive symptoms, but the relationship between mental disorders and endometriosis is being observed in other studies. Thus, when diagnosed with endometriosis, the patients should be evaluated by a multidisciplinary team, composed by the gynecologist, psychologists and psychiatrists, being investigated in relation to the possible existence of depressive symptoms, their diagnosis and treatment.

\section{Conclusion}

In patients with endometriosis there appears to be a tendency for mild depression, but there is no association with symptoms of moderate or severe depression.

\section{Statement}

In accordance with chapter XI I.2 of Res. CNS 466/2012, the registration number referring to the Certificate of Presentation for Ethical Assessment (CAAE): 31288914.5.0000.5243, approved by the Ethics and Research Commission of the Faculty of Medicine of the Universidade Federal Fluminense-FM/UFF/HU on 08/08/2014.

\section{References}

[1] Jansen, R.P.S. and Russell, P. (1986) Nonpigmented Endometriosis: Clinical, Laparoscopic, and Pathologic Definition. American Journal of Obstetrics and Gynecology, 155, 1154-1159. https://doi.org/10.1016/0002-9378(86)90136-5

[2] Berbel, B.T., Podgaec, S. and Abrão, M.S. (2008) Analysis of the Association between the Clinical Situation Reported by Patients with Endometriosis and the Site of Disease Involvement. Journal of Medicine, 87, 195-200.

[3] D’Hoo Ghe, T.M. and Hill, J.A. (2008) Endometriosis. In: Berek, J.S.N., Ed., Treaty 
of Gynecology (Chapter 29), Guanabara Koogan, Rio de Janeiro, 456-470.

[4] Eskena Zi, B. and Warner, M.L. (1997) Epidemiology of Endometriosis. Obstetrics and Gynecology Clinics of North America, 24, 235-258. | https://doi.org/10.1016/S0889-8545(05)70302-8

[5] Moen, M.H. and Stokstad, T. (2002) A long-Term Follow-Up Study of Women with Asymptomatic Endometriosis Diagnosed Incidentally at Sterilization. Fertility and Sterility, 78, 773-776. https://doi.org/10.1016/S0015-0282(02)03336-8

[6] Chen, L.C., Hsu, J.W., Huang, K.L., Bai, Y.M., Su, T.P., Li, C.T., Yang, C., Chang, W.H., Chen, T.J., Tsai, S.J. and Chen, M.H. (2016) Risk of Developing Major Depression and Anxiety Disorders among Women with Endometriosis: A Longitudinal Follow-Up Study. Journal of Affective Disorders, 190, 282-285. https://doi.org/10.1016/j.jad.2015.10.030

[7] Turner, J. and Romano, J. (1990) Cognitive-Behavioral Therapy. The Management of Pain. Lea \& Febeger, Philadelphia.

[8] Lorenzatto, C., Vieira, M.J.N., Marques, A., Benetti-Pinto, C.L. and Petta, C.A. (2007) Evaluation of Pain and Depression in Women with Endometriosis after Multiprofessional Group Intervention. Journal of the Brazilian Medical Association, 53, 433-438.

[9] American Psychiatric Association (2000) Manual of Diagnosis and Statistics of Mental Disorders. 4th Edition, Medical Arts, Porto Alegre.

[10] Bromet, E., Andrade, L.H., Hwang, I., Sampson, N.A., Alonso, J., Of Girolamo, G., De Graaf, R., Demyttenaere, K., Hu, C., Iwata, N., Karam, A.N., Kaur, J., Kostyuchenko, S., Lépine, J., Levinson, D., Matschinger, H., Mora, M.E.M., Browne, M.O., Posada-Villa, J., Viana, M.C., Williams, D.R. and Kessler, R.C. (2011) Cross-National Epidemiology of DSM-IV Major Depressive Episode. BMC Medicine, 9, 90-106. | https://doi.org/10.1186/1741-7015-9-90

[11] Eriksen, H.L.F., Gunnersen, K.F., Sørensen, J., Munk, T., Nielsen, T. and Knudsen, U.B. (2008) Psychological Aspects of Endometriosis: Differences between Patients with or without Pain on four psychological variables. European Journal of Obstetrics \& Gynecology and Reproductive Biology, 139, 100-105. https://doi.org/10.1016/j.ejogrb.2007.10.002

[12] Evans, R.G., Barer, M.L. and Marmor, T.R. (1994) Why Are Some People Healthy and Others Not? The Determinants of Health of Populations. Aldine de Gruyter, New York. 